\title{
DIETA SENSORIAL
}

\section{Programa de actividades cotidianas basado en la teoría del procesamiento sensorial}

Laura Álvarez de B., Mg. Ed., TOL*

Este articulo presenta una revisión sobre los principios de los programas basados en el concepto de desarrollo y procesamiento sensorial, particularmente, los propuestos como medida de manejo integral al infante con y sin limitación funcional o problemas de interacción. A partir de ellos, se plantea un programa cotidiano relacionado con la experiencia en el medio colombiano.

Dentro de la teoría del procesamiento sensorial de revisan los antecedentes para comprender la pertinencia y poder de las cualidades sensitivas en la actividad humana. Ayres (1972, 1974,1979) fue la pionera de la teoría de integración sensorial, su aporte fue valioso para la comprensión del procesamiento sensorial inherente a las ejecuciones humanas. Esta autora vinculó a las teorías del neurocomportamiento el uso de la sensación como base de la actividad, y creó un tratamiento para niños con desórdenes del aprendizaje y el comportamiento. Resaltó la importancia e influencia de la interpretación, procesamiento y respuesta sensorial en el desarrollo típico del niño. Estudió extensivamente los sistemas táctil y vestibular y demostró la necesidad de reconocer tempranamente sus funciones y repercusiones en el desempeño y comportamiento humano.

La terapia de integración sensorial ha sido practicada en terapia ocupacional por treinta años, y a partir de los aportes de Ayres, se ha venido ampliando su conocimiento y desarrollando múltiples estrategias para la atención a diferentes poblacio-

- Profesora Asociada Universidad Nacional de Colombia. Bogotá. 
nes (Deams, 1994; Ottenbacher \& Shart, 1985; Faber, 1982; Sweeney, 1986; DeGangi \& Greenspan, 1988, 1991; Cermak, 1988; Fisher, Murray \& Bundy, 1991; Kimball, 1990; DeGangi, 1991; Koomar \& Bundy, 1999; Colby, Laurel \& Windeck, 1993; Cermak, 1991; Oetter, Richter \& Frikc, 1995; Reisman \& Hanschu, 1992; Kinnealey \& Wilbarger, 1993; Dunn, 1994; Wilbarger \& Wilbarger; 1995; Lai Fischer/ Magalhaes, 1996; Odergren, Iwasaki \& Forssberg, 1996, Lissy, 1997; Williams \& Shellenberg, 1997).

Dentro de los avances más significativos en la teoría de integración sensorial se cuentan la reestructuración del procedimiento diagnóstico, reconocido actualmente como Test de integración sensorial y praxis (Ayres, 1989) y la consecuente variación en los factores de análisis; la mayor precisión y profundidad en las proposiciones teóricas de plasticidad cerebral; el proceso secuencial de desarrollo en espiral; y la jerarquización de funciones del SNC en relación con la organización sistémica (Short-De Graff, 1988; Fisher \& Murray, 1991; Kielfhoffner, 1991). Estas teorías también ha sido expandidas por las neurociencias que han incluido los aspectos relacionados con el procesamiento sensorial en los estudios mente-cuerpo como lo reporta la literatura (Gorbach, Triscalov, Kiznetsovaa \& Shevelev, 1989; Kandel, Schwartz, \& Jeseel, 1991; Murphy, 1993; Ornstein \& Sobel 1987; Zuckerman, 1994; Lezak, 1995; Greenspan, 1997).

A pesar de que esta no es una revisión exhaustiva, se encuentran gran variedad de investigaciones que validan el uso y eficacia de los enfoques sensoriales, tanto en la remediación como en la prevención y promoción del crecimiento personal, la literatura pública da cuenta del efecto de los programas relacionados con la actividad sensorial en una variedad de poblaciones (Magnum, Ottenbacheer, McCue \& Keefe, 1981; Wilbarger, 1971-1984; Kuharski, Res, Cook \& Guess, 1985; Yack, 1989; Kimball, 1990; Oliver, 1990; Cermak, Trimble,Coryell \& Drake, 1990; David, 1990; Cermak, Morris \& Koomar, 1990; Law, Polatjko, Schaffer, Miller \& Magnab, 1991; Scheneider, Kraemer \& Suomi, 1991; Reisman \& Hanschu, 1992; Colangelo, 1992; Lightsey, 1993; Richter \& Polatajko, Law, Janice, Schajjer \& Magnab, 1991; Maylloux, 1992-1993; Koomar \& Friedman, 1992 a-b; Miller \& Kinnealey, 19931994; Trott, Laurel \& Windec, 1993; Tseng \& Cermak, 1993; Willams \& Shellenberger, 1994; Willoughby \& Polatojko, 1994; Dunn, 1994; Neistadt, 1994; Wright \& Sugden, 1996; Lissy, 1997).

En general los estudios reportan los efectos de la estimulación de uno o varios sistemas sensoriales y el alcance de las respuestas adaptativas después de someter 
a las personas (niños y adultos) a programas estructurados en ambientes propicios para el desarrollo y procesamiento sensoriomotor.

\section{El concepto de procesamiento sensorial}

Los principios relacionados con la teoría del procesamiento sensorial son con frecuencia difíciles de explicar en palabras simples. El procesamiento sensorial, es el mecanismo de auto-organización neurobiológica resultando de la interacción entre la maduración del SNC, la identificación los impulsos sensoriales provenientes del medio interno y externo, y la respuesta o comportamiento de las personas a las demandas del medio ambiente.

En otros términos, el procesamiento sensorial es el mecanismo primario de organización del mundo externo, a partir de la representación mental interna. El tratar de comunicar algunas de estas complejas teorías del neurocomportamiento, tanto a profesionales de rama ajenas a la salud, como a los padres y cuidadores infantiles, no ha sido una tarea fácil. (Wilbarger, 1971-1984), utilizó el término dieta sensorial planteándolo en paralelo con la dieta nutricional como metáfora para explicar las ideas claves de un programa basado en un eficaz desarrollo y procesamiento sensorial, debido a que la gente comprende fácilmente la esencia y principios de la dieta nutricional. En este sentido, para resaltar la importancia y regularidad que implica el plan sensorial diario, lo relaciona con al necesidad de las tres comidas al día y dos meriendas intermedias. El concepto de complejidad y multiplicidad de factores que intervienen en la planificación de una dieta nutricional, se aplica también a la dieta sensorial.

En síntesis plantea que las necesidades de las dietas sensoriales y nutricionales son similares. Son muchos los autores, que reconocen que el ser humano necesita una correcta combinación de impulsos sensoriales para alcanzar un nivel óptimo de despertar/excitación y desempeño (Wilbarger \& Wilbarger, 1995; Zucherman, 1994). Los terapeutas Ocupacionales estudiamos la necesidad y significado de las actividades sensoriales en el desarrollo humano, por lo que se considera indispensable la planeación de tiempo - espacios - composición - dosificación, de ellas a lo largo del día, la semana, la edad y caracterísiticas del usuario. Estudios relacionados con el efecto del tiempo/experiencia sensorial, han considerado la semejanza de algunas actividades cotidianas con la merienda de la dieta nutricional, porque en ellas influyen las diversidades sensitivas de las personas, su predisposición personal, el 
nivel sensitivo/sensorial: hipersensible, hipo sensible, fluctuante; los periodos de tiempo, entre otros.

En este sentido, se deben diseñar las dietas sensoriales de acuerdo a las modalidades de intervención dentro de las cuales se mencionan: dietas genéricas para programas de promoción, es decir para la población "normal" sin riesgo o limitación; dieta a corto plazo para programas de prevención, es decir, para poblaciones a riesgo social-ambiental-o biológico; $y$ otras dietas a largo plazo han sido diseñadas para tener efectos más duraderos sobre el comportamiento y el desempeño, y corresponden a los programas de remediación.

La prescripción significativa de una dieta sensorial es función del terapeuta ocupacional y parte de la identificación de diversidad individual de necesidades de un programa cotidiano de desarrollo y procesamiento-sensorial, el cual debe partir de la observación detallada đel desempeño de la persona tanto en su actividad usual (trabajo- estudio; juego-diversión; manejo personal) como en actividades ajenas al trabajo formal, que con relativa frecuencia presentan algunas personas durante la ejecución de acciones sin propósito tales como: movimientos nerviosos en el asiento, movimientos persistentes de los dedos, acciones constantes de masticar el lápiz, etc. que demandan para su procesamiento acciones inmediatas de gran efecto sensorial que tiene como finalidad cambiar el foco de atención (por ejemplo; pedir alcanzar o manipular objetos, soplar o inflar bombas, hacer reír, gesticular, frotar, etc), es decir, pedir una actividad corta, con propósito, dentro de las actividades formales de trabajo, estudio o cuando se presente el comportamiento, estos son los denominados snack sensoriales.

El uso de la sensación para mantener el desarrollo y el comportamiento típico ha sido extendido para tratar a personas que tiene comportamiento atípico

La orientación de un programa de interacción de la persona con los impulsos sensoriales internos (los del propio cuerpo) y externos (los del medio ambiente) a lo largo del día, debe ser analizado a partir de la conciencia que cada individuo posee sobre la oportunidad y la duración de la actividad; el conocimiento acerca de la intensidad del evento sensorial; y la comprensión del efecto del impulso sobre la gran variedad de neuromecanismos esenciales para la organización del comportamiento adaptativo (Milkman \& Sunderwirth, 1987; Wilbarger \& Wilbarges, 1995; Williams \& Shellenberger, 1992; Zuckerman, 1994). 
En otras palabras el programa debe estructurarse sobre el nivel particular de procesamiento que posee la persona y no sobre el comportamiento o procesamiento que el adulto (padre, maestro, jefe) espera de acuerdo a la norma o comportamiento típico. El programa cotidiano o dieta sensorial, intenta lograr y mantener niveles óptimos de alerta o despertar para las ejecuciones personales, seleccionando cuidadosamente la actividad sensorial básica. Cada persona tiene necesidades sensoriales únicas (Wilbarger \& Shellenberger, 1994; Zuckerman, 1991-1994). Algunas necesidades son sutiles, otras son más intensas (Milkman \& Sundeerwirth, 1987). Se supone entonces que son las gratificaciones sensoriales las que despierten en las personas intereses particulares, de hay que el trotar, escalar, practicar el paracaidismo y otras gran variedad de actividades son interpretadas como formas de búsqueda y satisfacción de emociones sensoriales. La interpretación de las necesidades sensoriales de los usuarios de un programa sensorial se relaciona con el análisis de los comportamientos inusuales de búsqueda, evasión o rechazo de sensaciones tales como persistencia de movimientos, ignorar o buscar el peligro, agrado/ desagrado al contacto físico, movimiento súbito, olores, sonidos, texturas, etc.

Por otra parte, el programa pretende encontrar significado a las sensaciones comunes, utilizando como referente el comportamiento típico. Existe suficiente evidencia sobre la contribución y efecto de una gran variedad de neuromoduladores y otros mecanismos complejos en las actividades físicas y sensoriales en general (Milkman \& Sunderwirt, 1987; Omstein \& Sobel, 1987; Reisman \& Hanschu, 1992; Zuckerman, 1994).

Se considera así mismo, que el desarrollo normal ocurre a partir de una gran cantidad de experiencias sensoriales seguras y/o gratificantes (Montague, 1989; Zubeck, 1979). Cuando la persona vivencia experiencias sensoriales atípicas, se presenta una interrupción o alteración en su comportamiento y desempeño. La deprivación sensorial o carencia de oportunidades ambientales de interacción, movimiento, manipulación, expresión etc, produce tanto desórdenes del comportamiento, como dificultades en el desarrollo (Zubeck 1979), además se ha visto leve perdida de su capacidad sensorial en la edad adulto reflejada en la disminución de la respuestas protectivas, primitivas, o comportamientos grotescos o extraños (Montague 1989). El reconocido stress ocasionado por efecto de carga sensitiva (Selye 1976). La auto-estimulación y los comportamientos auto injuriosos observados en las instituciones de cuidado son con frecuencia el resultado de un pobre desarrollo o insufi- 
ciente procesamiento sensorial. Por tanto, dichos comportamientos requieren de corrección, y las dietas sensoriales son una alternativa efectiva.

En este sentido se han desarrollado varias alternativas para la observación detección y/o seguimiento de los niveles de procesamiento sensorial relacionados con el desarrollo y el comportamiento típico. Entre los más recientes están; un inventario para detectar demoras en el desarrollo de Reisman y Hanschu (1992) en el que describe comportamientos de búsqueda o evasión sensorial dentro de las ejecuciones de la vida diaria; un instrumento "Screening" para padres de DeGangi (1995) con un chequeo de ayuda; el perfil de aprendizaje temprano de Stephanie Park (1994); el perfil de desarrollo del niño de Bay Cove, Dochesther dentro de su plan de servicio a la familia y el programa de intervención temprana (1996); el cuestionario de desarrollo y procesamiento sensorial Cermak y Miller (1991), ya validado en Colombia, el inventario sensorio motor de Dunn (1991); el chequeo de Desordenes de la regulación de Abby Chang (1994).

\section{La aplicación del concepto de Programa de Dieta Sensorial}

El concepto sobre la utilidad del procesamiento sensorial para promover o mantener los comportamientos normales, puede extenderse para tratar las personas que tienen comportamientos atípicos. La actividad sensorial puede mejorarse con el diseño y ejecución de un programa cotidiano de procesamiento sensorial. Su propósito primario es ayudar a la persona a lograr y mantener un umbral de excitación óptima, según afirma Wilbarger \& Shellenberger, 1992, o para proveer un impulso sensorial uniforme e intenso que produzca un cambio en la capacidad de procesamiento sensorial Wilbarger \& Wilbarger, 1995.

Los componentes de un programa de dieta sensorial pueden ser usados como una guía para comprender las influencias sensoriales tanto en el comportamiento típico como el comportamiento desordenado. Es justamente sobre el significado de las bases sensoriales del comportamiento, que se hace posible el desarrollo de programas, tanto para modificar los comportamientos atípicos, como para promover el desarrollo normal del infante. La planificación adecuada contribuye al proceso típico del desarrollo de cualquier persona, y reviste especial importancia para aquellas que presentan una interrupción, desorden o capacidad disminuida en el procesamiento sensorial, a tal nivel, que compromete su desempeño en las actividades. Un programa sensorial debe incorporar todos los aspectos de la vida diaria, por 
tanto incluye un componente particular para el hogar, en especial cuando los problemas de procesamiento sensorial, son dependientes de discapacidades físicas o psicológicas. En este sentido su estructura debe responder a la atención eficiente del adulto y el niño, dentro del proceso de atención adecuada y de remediación del desempeño, no solo como complemento del programa de atención individual sino como parte integral de este, es decir, para apoyar e impulsar el desarrollo de la persona en todo acto cotidiano. En el caso de infantes, en particular cuando se encuentran institucionalizados desde temprana edad, la dieta sensorial aplicada por cuidadores es esencial para promover su desarrollo, al igual como medida de prevención, para cuando existe riesgo biológico, ambiental o social.

\section{Principios generales utilizados para el desarrollo de un programa sensorial}

Las complejas teorías de neuro-comportamiento, las neurociencias y las que subyacen en la planificación de éste enfoque de actividades van más allá del alcance de esta presentación, debido a que el diseño de un programa de dieta sensorial adecuado para cada.

Diseñar un programa sensorial es elegir el tipo, cualidades, efecto regulador, duración e intensidad de la actividad

tipo diferencial de procedimiento sensorial, pretende contribuir a la modificación o desarrollo de las estructuras del SNC cuyo efecto puede ser observado en el cambio de comportamiento. Se requiere, por tanto, un análisis profundo para la elección de actividades. Este incluye el tipo y cualidades de la actividad, su efecto regulador (exitatorio o inhibitorio), la duración e intensidad de la sensación, la selección y disposición del ambiente con posibilidades de modificación de acuerdo a necesidades, y las particularidades de jerarquización, juzgando que factores son más importantes para el caso y momento.

Este proceso es similar al propuesto en los programas para el desarrollo del pensamiento lógico en el que lo significativo no es-afirmar o negar sobre el postulado, sino sopesar la decisión. Es decir, no se trata de elegir la actividad para un fin determinado, sino sopesar las respuestas adaptativas alcanzadas por la persona - para así determinar el curso del programa sensorial. 
Innovaciones recientes al respecto reflejan la necesidad de créar programas cada vez más concretos y específicos para las diferentes áreas de intervención y tipos de procedimientos sensoromotores. Dentro de estos se encuentra el de Wilbarger y Wilbarger, 1991 sobre desórdenes en la modulación sensorial, demostrando la necesidad e importancia de apoyar la intervención terapéutica con programas domésticos específicos. El trabajo de Oetter, 1995 nos alerta sobre la importancia de la sincronización de la succión- masticación-respiración, para el desarrollo de mu: chos aspectos del desempeño humano. El trabajo de Richter y Frick, 1995 describe como la modulación y excitación de las funciones motora- oral- respiratoria y visual, subyacen a la adquisición de destrezas más complejas. Reisman y Hauschu, 1992 describen gran variedad de comportamientos sensoriales presentados en personas con incapacidades en el desarrollo resaltan los de evasión, búsqueda, autoestimulación y excentricidad y los incluyen con puntos sensoriales cunatificados dentro de su inventario.

Paralelamente se han elaborado programas de dieta sensorial tanto en el área clínica como en el sector escolar, dentro de los que cuentan el de prevención o alerta, desarrollado por Williams y Shellenberger, 1992 y usado en la escuela Gallup, Nuevo México, para ayudar a los niños a aprender acerca de su propia atención y estado de excitación o despertar, en el cual se diseñaron unas estrategias de trabajo que dieron como resultado que los niños comenzaron a entender y controlar su auto- regulación. El programa utilizó los principios de la investigación sensorial e involucró la nivelación cognoscitiva a partir de experiencias sensoriales. Fue desarollado con todos los niños de un salón de clases produciendo resultados impresionantes, fue considerado un sistema eficaz y de bajo costo, que demostró la importancia del programa de procedimiento sensorial.

\section{Planeación de la dieta "programa sensorial cotidiano"}

La construcción de un programa cotidiano que abarque al máximo los componentes del procedimiento sensorial envuelve dos dimisiones la atención directa a la persona o grupo y la consecuente atención indirecta u orientación a la familia, cuidador maestro que esta en contacto prolongado con el usuario. Para garantizar la efectividad del programa deben considerarse los aspectos de remisión, consulta y compromiso; evaluación-orientación; y razonamiento critico sobre lo reportado en la historia sensorial. 


\section{Remisión, consulta y prescripción}

El diseño de un programa sensorial se determina por el tipo especifico de problema presentado, así como del propósito y la ubicación de la intervención. El primer paso, obviamente, en cualquier proceso de intervención es contar con la colaboración del niño o persona a la que va dirigido el proceso, sus intereses, metas y percepciones; el reconocimiento sobre la importancia y veracidad de la información y sus implicaciones; la aceptación de las actividades y su ejecución tanto por el equipo familiar como por todos los miembros de la comunidad. Hanft, 1989 presenta un excelente modelo centrado en el cuidado paciente-familia, apoyado en una serie comprensiva de protocolos que tienen que ser diligenciados por el terapeuta ocupacional para reunir información general acerca del usuario, y de ésta, partir al diseño y control del programa de intervención.

El plan de actividades que se desprende de la atención terapéutica de calidad es complejo e intensivo y debe partir del conocimiento amplio y especifico sobre las teorías del procesamiento sensorial. Las actividades propuestas son en apariencia simples, pero poseen efectos poderosos y duraderos sobre el comportamiento y la fisiología humana. Es necesario un entrenamiento especifico para entender que las estrategias de intervención involucran una serie de precauciones, supervisión y control cercano por parte del terapeuta o profesional previamente capacitado.

\section{Evaluación-orientación}

La ejecución directa de un programa basado en el desarrollo y procesamiento sensorial significa un compromiso y un contrato especial con cada uno de nuestros usuarios y sus familias. El primer paso en la intervención comienza con la evaluación del comportamiento, lo que también es un proceso de formación permanente tanto a maestros como a familia y comunidad. Se hace necesario compartir (con otras personas menos expertas en el tema) las teorías que utilizamos para interpretar los comportamientos sensoriales básicos, a partir de los cuales pretendemos enriquecer la particularidad de cada procesamiento sensorial. Debe entonces lograrse la comprensión del proceso por parte del usuario (en relación con sus posibilidades de comprensión) y de la familia y maestro. Esta no es una tarea fácil, pues la mayoría de las razones que nosotros buscamos son respuestas o evidencia de experiencias sensoriales subconscientes, es decir, reacciones o comportamientos que evidencia la persona, de los que no es consciente o por lo menos no pensó o midió sus 
consecuencias. ejemplo: ante un contacto suave, desprevenido, el niño reacciona agresivamente; personas que se aíslan o alejan ante sonidos fuertes u olores determinados; rechazo a cierto tipo de texturas o ropas, etc. La identificación de estos comportamientos son parte central del proceso evaluación / orientación. Buscar que los usuarios y sus familias comprendan la relación entre los síntomas y:la intervención es una conclusión importante del proceso evaluativo.

Razonamiento crítico del caso

Comprende dos momentos: la entrevista y el análisis e interpretación de diversas interacciones con la persona, su familia y su medio.

La entrevista: La efectividad de una entrevista clínica, escolar o personal, depende de la forma como se oriente la relación de los comportamientos sensoriales con el desempeño cotidiano de la persona. En este programa, es a partir de una entrevista interactiva que se contrasta la téoría del procedimiento sensorial con el comportamiento descrito. De esta manera, lo reportado en la historia no solamente reúne información sobre el sujeto, sino que contribuye a una comprensión de las cualidades particulares de su comportamiento y desempeño, llegando a una comprensión más profunda del rol del procesamiento sensorial y por ende, a la selección más acorde de los objetos, ambientes, interacciones, $y$ comportamientos adaptativos organizantes o desorganizantes del usuario. Los siguientes son algunos elementos que considero debe contener la entrevista:

- Indagar acerca de los comportamientos, asociados con cada uno de los sistemas sensoriales. La elección de la respuesta a las experiencias cotidianas incluye el tocar/táctil; movimiento/vestibular/propiocepción; control motor/oral en la alimentación; respiración; visión; audición; olfato y gusto.

- El interrogatorio deberá incluir la discusión acerca de la excitación o niveles de actividad. ¿Por ejemplo, a que hora o período del día la persona desempeña o actúa organizadamente? ¿Cuándo presenta el comportamiento más desorganizado? ¿Modifica el nivel de excitación o actividad ante el incremento de estímulos ambientales; lo aumenta o lo disminuye? ¿Bajo que condiciones, la persona se muestra más segura o con mejor balance de los niveles de excitación y actividad? ¿Hay ciclos predecibles de organización/desorganización del comportamiento? 
- Pueden identificarse actuaciones predictivas o de búsqueda sensorial. Frecuentemente estos son indicios de lo que la persona hace en su proceso personal de auto-organización. Estas pueden aparecer con pocas evidencias, con frecuencia ser ignorados, no ser entendidos o ser descritos de manera poco clara.

- Indague sobre la vigencia de los comportamientos, si se presentaron en el pasado, o en la infancia. Esto puede explicar con mayor certeza la evolución de los comportamientos $\mathrm{Ej}$.: busque información sobre los cambios presentados en algunos síntomas de desorden en el procedimiento sensorial, si desaparecieron o reaparecieron.

- Describa las actuaciones de dos días típicos de la cotidianidad y analice las cualidades sensoriales que encontró. Estos son comúnmente un día de escuela o trabajo y un fin de semana $u$ otro día no estructurado $¿ E j .:$ describa lo primero que le gusta hacer a la persona cuando se levanta, o cuando tiene que ir a dormir, y que textura prefiere en sus sabanas, pijamas $u$ objetos que toma para dormir. Trate de ilustrar lo visual, lo sonoro, las actividades, y las interacciones que la persona puede encontrar a lo largo del día.

El análisis e interpretación de las interacciones. La permanente correlación entre los comportamientos descritos, observados y contrastados con la teoría del procedimiento sensorial dan como resultado final el poder aproximarse a un programa general de actividades dentro de la cotidianeidad de la persona con cualidades, ambientales, transiciones $e$ interacciones que respondan al nivel actual de procedimiento y que son reflejo de la maduración e integración sensorial. Esto provee un cuadro sensorial vigente para cada persona en relación con sus intereses creencias, y valores.

Se utilizan también cuestionarios escritos para dichos procesos. Muchos de ellos se han organizado en forma de historia sensorial con una buena base de preguntas. Reisman \& Hanschu, 1992. Sin embargo los cuestionarios no son los suficientemente precisos para determinar por sí mismos la valoración del procesamiento sensorial. La información obtenida en la forma escrita es muy importante pero deber se lo mas completa y precisa posible. Sin embargo, se requiere de un cuidadoso trabajo para extraer la descripción de comportamientos que al lector le puede parecer insignificantes. Nuestros usuarios y/o sus cuidadores, a veces piensan que 
los síntomas inusitados son típicos y no los mencionan o no les dan el mérito suficiente. Por el hábito, ellos pueden ignorar o pasar desapercibidos los impulsos desorganizan tez, y pueden ser inconsecuentes e incluso distorsionar la percepción sensorial.

\section{Plan de actividades}

Cada persona tiene necesidades sensoriales únicas y diferenciales. El terapeuta ocupacional trata de construir un plan de trabajo alrededor de estas necesidades, de los recursos disponibles (incluyendo el tiempo, dinero y espacio), y de las preferencias y estilos personales. Wilbarger, 1984 plantea que la construcción del plan o dieta sensorial se asimila más a la elección del menú que al seguimiento de una receta. En este sentido se deben considerar ciertos aspectos relevantes:

- Orientación temporal de las rutinas. La experiencia clínica ha mostrado que ciertas actividades tienen efectos moduladores sobre el sistema nervioso en ciertos períodos de tiempo, y que diferentes experiencias sensoriales son más efectivas que otras. Las actividades que incluyen presión táctil profunda y los impulsos vestibulares y propioceptivos, se piensa que tienen efectos más profundos sobre el comportamiento. Los ejemplos incluyen jugar con un equipo de parque o en un "centro de juego", practicar aeróbicos, Saltar sobre un trampolín o jugar a la lucha. Otras actividades pueden utilizarse para cambiar los estados o modificar el humor e incluyen experiencias táctiles, visuales, auditivas, olfativas y aportes orales y respiratorios (Frick, 1992; Oetter et al., 1995) con juguetes para agitar y manipular (McClannahan, 1990), incluye meriendas y ciertos tipos de música.

Las actividades deben ser organizadas cuidadosamente en el tiempo, es decir, distribuidas durante todo el día para ayudar a la persona a alcanzar y mantener un óptimo estado de excitación / inhibición durante las horas de vigilia sueño. El afinamiento sensorial puede ser programado en horas claves del día, tal como la primera actividad que se hace en la mañana, en el periodo de transición, en las horas de la tarde y en la noche. Algunos de los cambios importantes en el sistema nervioso pueden provenir del conocimiento que se tenga sobre cómo se pueden cambiar las actividades para alcanzar efectos duraderos. La experiencia clínica ha mostrado que los efectos del movimiento específico de actividad vestibular pueden permanecer en el organismo de $4 \mathrm{a}$ 
8 horas, mientras que las actividades que involucran acciones en todo el cuerpo, tracción articular, contracción, actividàd muscular fuerte y ciertos efectos respiratorios tienen generalmente dos horas de duración. Wilbarger desarrolló un protocolo especifico de intervención en el que integra la presión táctil profunda y la comprensión articular identificando que sus efectos duran de 1 a 2 horas. Igualmente se ha demostrado que los impulsos visuales y auditivos pueden cambiar el estado de excitación, desencadenar respuestas emocionales, y actuar sobre el sistema de memoria, pero sus éfectos no son duraderos a menos que sean usados muy específicàmente.

- Las rutinas. La consistencia en las rutinas contribuye al desarrollo de las actividades de la vida diaria tales como levantarse, vestirse, comer, bañarse $y$ dormirse a tiempo y uso adecuado de los periodos de transición. Las rutinas especificas se desarrollan para ayudar a la persona a mejorar su nivel de atención y alerta en la escuela o trabajo. Existen protocolos terapéuticos que orientan la preparación para la comida; la disposición de la cavidad oral para comer; la orientación de la distribución o uso del tiempo en cada tarea a fin de establecer el ritmo personal y modificarlo de acuerdo a las demandas del medio ambiente.

La regulación de las rutinas en concordancia con las expectativas del medio; es un factor esencial en el desempeño individual, pues estas se han constituido en síntoma central de remisión y/o calificación del desarrollo personal

Esto no quiere decir que se desconozcan las diferencias individuales, sino por el contrario, que se reconozca el nivel de procesamiento propio.

- Las interacciones. Se les puede aconsejar o recomendar a los padres y cuidadores, formas especiales de interacción que provean sensaciones apropiadas, por ejemplo, uno podría modificar las cualidades de la voz: intensidad y tono, la cantidad y proporción de habla; el apoyo a estímulos auditivos con ayudas visuales; el uso del contacto personal previo o durante la instrucción oral, etc.

Las cualidades de contacto personal o táctil, pueden perfeccionarse y complementarse con elementos y adecuaciones al medio. En otros casos es necesario reducir o cambiar las demandas de contacto visual. La camaradería y com- 
portamiento alegre durante las experiencias sensoriales pueden ser altamente motivante logrando que la acción sea más integradora. Sin embargo algunas actividades requieren de supervisión o apoyo directo, lo que hace que el terapeuta esté no solo participando directamente en el contacto cotidiano de la persona, sino además de orientar, seguir y controlar el plan indirecto.

- La estructura de ambiente. En el ambiente cotidiano se puede ubicar algún equipo especial en el sitio donde se desarrollará el programa a fin de fomentar la auto-selección y auto-dirección de la actividad. La exploración directa es una de las más poderosas y efectivas formas de alcanzar respuestas adaptativas. Esto debe permitir a la persona comprometerse con la experiencia sensorial. Actividades que involucren las acción muscular resistida; compresión y tracción articular; movimientos en diferentes planos especiales, posiciones corporales y superficies; manipulación y contacto corporal con variedad de texturas $y$ temperaturas, son entre otras experiencias de apoyo al procesamiento sensorial. Los espacios que le permiten a la persona sentirse segura y protegida, los ambientes sensorialmente controlados o sin restricción identificados o creados. Uno debería atender también a los elementos sensoriales del ambiente y en lo posible, eliminar o reducir que estímulos disociadores y mejorar la organización de los elementos según necesidades de cada usuario. En particular el sonido, la iluminación, el fondo visual y los olores deberán analizarse como aspectos positivos o negativos para la persona, y adaptarse según necesidades. Si las anteriores son consideraciones para el ambiente cotidiano de interacción, para la atención directa o individual, estas condiciones deben ser más peculiares.

Las actividades no estructuradas. En todo programa sensorial, se hace necesario el desarrollo y/o mantenimiento de las destrezas para el uso natural del tiempo no estructurado. Las actividades aquí desarrolladas tienen grandes beneficios sensoriales. Es en este espacio, en el que la persona debe elegir la actividad a realizar.

Con frecuencia, ante comportamientos típicos o atípicos, por ejemplo, la persona elige la televisión a la actividad física. Conocedores de la importancia de esta última (en los comportamientos típicos) y la incidencia y necesidad (en los comportamientos atípicos) su clarificación se logrará a partir de ambientes experimentales con actividades que sean los suficientemente llamativas a acordes a la necesidad 
individual, que logren la auto-iniciación, la plena satisfacción y el propósito en la acción.

\section{CONCLUSIÓN}

Un programa cotidiano de actividades basado en el procesamiento sensorial, es una constante combinación de experiencias gratificantes para cada persona. La analogía planteada por Wilbarger, 1995 entre los patrones nútricionales y su forma de análisis: el colesterol, los antioxidantes etc., son cuantificados en números o cifras representativas. El programa sensorial debe ser analizado con igual complejidad y profundidad, y es, en éste sentido, que se requiere del análisis cada vez más profundo y sistemático de los factores representativos del procesamiento sensorial en niños y adultos, a fin de lograr el establecimiento de parámetros cuantitativos y cualitativos de dichos comportamientos. La última década ha ofrecido información sustancial sobre los neuropéptidos, que pueden ayudarnos a entender el poder holístico de la actividad.

El trabajo de Pert, y sus colaboradores, los de Rossi y Creel, 1994 citado por Wilbarger, $y$ los otros citados anteriormente, han profundizado significativamente en la comprensión de la relación sensación-cuerpo-mente. Estas discusiones en las neurociencias, surgen contemporáneamente a la utilización de las bases teóricas de la integración sensorial en las experiencias clínicas de los terapeutas ocupacionales.

\section{REFERENCIAS}

Ayres, J. (1972). Sensory integration and learning disorders. Los Ángeles: Western psychological services.

Ayres, J. (1974). The devolpment of sensory integrative theory and practice: A collection of the works of Jean Ayres. Rockville, M.D.: American Occupational Therapy Association.

Cermak, S., Morris, M., \& Koomar, J. (1990). Praxis on verbal command and imitation. American Journal of Occupational therapy, 44 (7), 641-645.

Daems, J. (1994). Reviews of reach in sensory Integration International. 
DeGangi, G. A., Berk, R. a \& Larsen, L.A (1980). The measurement of vestibules based functions in preschool children. American Journal of Occupational therapy, 34, 452-459.

Dunn, W., (1994). Performance of typical children on the sensory profile: $\mathrm{Zn}$ item analysis. American Journal of occupational therapy , 48(11), 967-974.

Koomar, j. y Friedman, B. (1992b). The hidden Senses. Your Muscle Sense. Rockville, MD: THE American Occupational Therapy Association. Neurorehabilitation: A multisensory approach.

Farber, S. (De) (1982). Philadelphia: Saunders.

Fischer, A., Murray, E y Bundy, A. (1991). Sensory integration theory and practice, Philadelphia: F. A. Davis.

Frick, S. (1992). Respiration tape (cassette recording). Boulder, CO: Bell curve Records.

Hafts; B. (1989). Providing family - centered occupational therapy services. Sensory Integration Special Interest Section Newsletter, 12, 1-3.

Kimball, J. (1990).The emphasis's on integration no sensory. American. Journal of Mental Retardation, 92, 423-424.

Kosco, B. (1993). Fuzzy thinking. The new science of escape. Lexington MA: Lexington Books.

Milkman, H., y Sunderwirth, S. (1987). Craving for ecstasy. The consciousness and chemistry of escape. Lexington MA: Lexington Books.

Montague, A. (1989). Touching: The human significance of skin. New York: Columbia University Press.

Murphy, M. (1993). The future of human nature. Los Ángeles: Jeremy P. Thatcher/ perigee.

Otter, P., Ritcher, E. y Frick, S. (1995). MORE. Integrating the mount with sensory and postural function. Llugo, MN: PDP press.

Oliver, B. (1990). The social and emotional issues of adults with sensory defensiveness. Sensory integration Special Interest. Section Newsletter, 13, 1-3. 
Ornstein, R., y Sobel, D. (1987). The heading brain. Breakthrough discoveries about how the brain Keeps us healthy New York: Simon \& Schuster.

Reisman, J., y Hanschu, B. (1992). Sensory Integration Inventory.-Revised for individuals with developmental disabilities. Llugo, MN: PDP press.

Richter, E. y Colangelo, E. (1992). Proceeding of the Sensory Integration Symposium

Tseg, M. y Cermak, S. (1993). Ergonomic and perceptualmotor aspects of handwriting. American Journal of occupational therapy, 47(10) 919-926.

Wilbarger, P. (1971). The identification, diagnosis and remediation of sensoriomotor dysfunction in primary school children. Title 111 ESEA project Report 5427. Goleta Union School District. California State Department Education.

Wilbarger, P. (1984). Planning and adequate sensory diet. Application of sensory processing theory during the firs year of life. Zero to three, 5(1).

Wilbarger, P. y Wilbarger, J. (1995). Sensory defensiveness and related social, emotional and neurological problems. Seminar presented in multiple locations. Denver, CO: Avanti Educational Programs.

Williams, M.S. y Shellenberger, S. (1992). An introduction to "How does your engine run?: the alert program for self- regulation. Albuquerque, NM: Therapy. Works.

Williams, M.S. y Shellenberger S. (1994). How does your engine run? A leader's guide in the Alert program for self regulation. Albuquerque, NM: Therapy Work.

Zubeck, J. P. (1979). Sensation deprivation. Fifteen years of research. New York: Appleton - century-crofts (now Appleton \& Lange, Norwak, CT).

Zuckerman, M.(1991). Psychobiology of personality. New York : Cambridge University Press.

Zuckerman, M (1994) Behavioral expressions and biosocial bases of sensation seeking. New York: Cambridge University Press. 